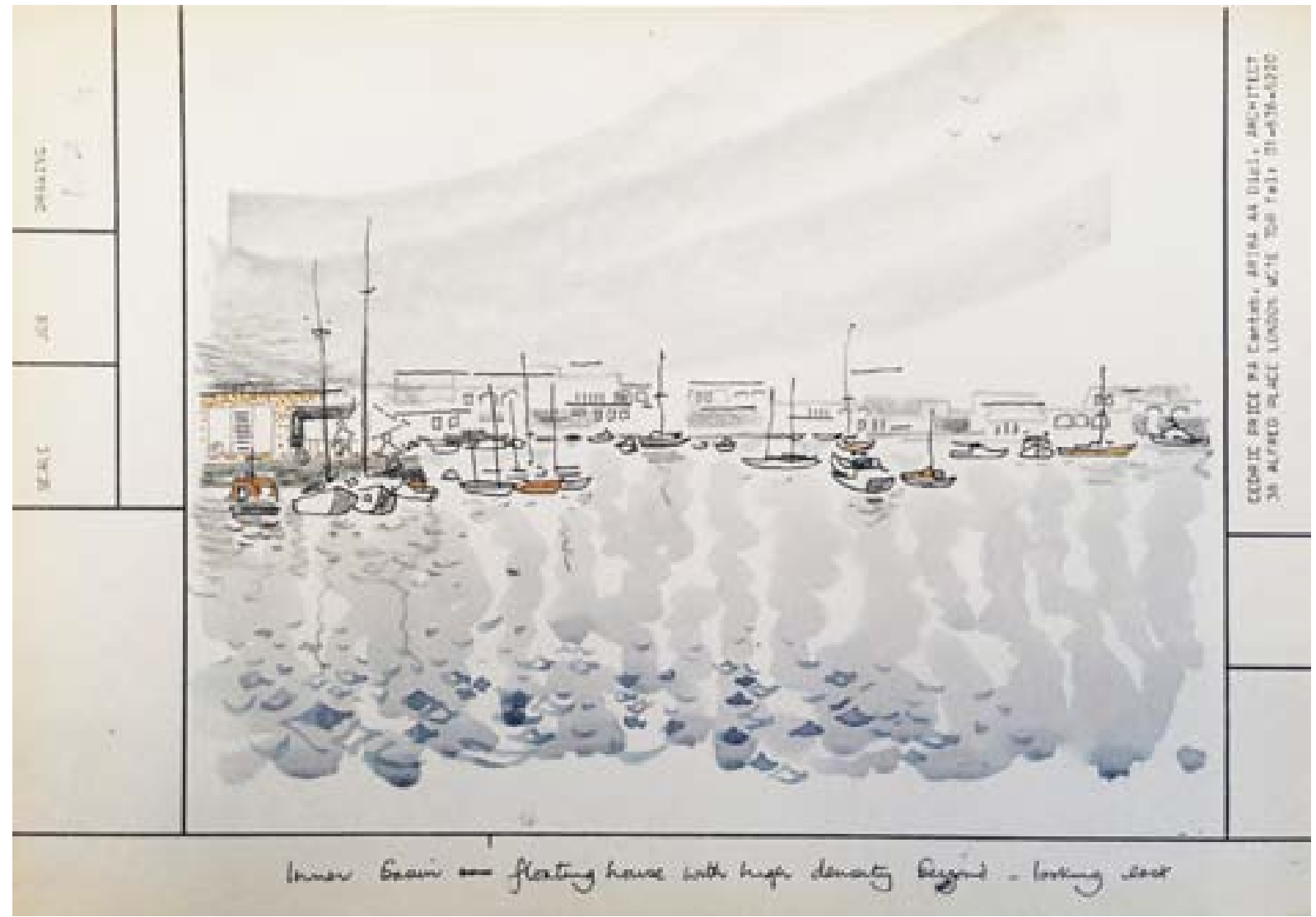




\section{'Landscapes with figures with gadgets': Lo pintoresco en la arquitectura experimental británica hacia 1970 Rodrigo de la $\mathrm{O}$}

Alrededor de 1970, Cedric Price y los miembros de Archigram solían presentar sus propuestas de hinchables, cúpulas geodésicas, robots o sistemas prefabricados formando parte de escenas paisajistas. El artículo esclarece la inserción de estos panoramas dentro de una tendencia histórica con fuerte significado cultural. Los historiadores Leo Marx y Reyner Banham lo conceptualizaron como la difícil búsqueda de un paisaje medio: un modelo ponderado y estabilizado por el arte, que desde el siglo XIX actúa bajo la creencia de que la máquina construirá un jardín.

\section{PALABRAS CLAVE}

Paisaje, tecnología, Cedric Price, Archigram, pintoresco

\section{KEYWORDS}

Landscape, Technology, Cedric Price, Archigram, Picturesque
DOS ÁRBOLES

Y NOMÁS
En noviembre de 1971 el arquitecto británico

Cedric Price comenzó a trabajar en un plan para adecuar un islote deshabitado en la desembocadura del Támesis, cerca de Southend-on-Sea, conocido como Two Tree Island'. Afrontaba una encomienda del empresario local David Keddie para construir un puerto deportivo, viviendas y espacios de ocio en línea con el crecimiento turístico que experimentaba entonces la región suroriental de Gran Bretaña. Un reportaje fotográfico tomado durante alguna de sus primeras visitas al lugar muestra un paisaje de marismas; un horizonte llano, solitario, parcialmente inundado y solo cubierto de herbáceas.

\author{
Manuel Rodrigo de la O Cabrera \\ Investigador postdoctoral en el \\ Departamento de Composición \\ Arquitectónica de la E.T.S.A.M. Obtuvo \\ el Premio Extraordinario por su Tesis \\ Doctoral, realizada con un contrato \\ predoctoral del programa internacional \\ PICATA-Campus Moncloa. Ha sido becario \\ José Castillejo en la Bibliotheca Hertziana- \\ Max Planck Institute for Art History en \\ Roma, academic guest en el Institute for \\ the History and Theory of Architecture del \\ ETH Zurich e independent scholar en el \\ Canadian Centre for Architecture. Entre \\ sus publicaciones se incluyen artículos \\ en Planning Perspectives, Change Over \\ Time, History of Gardens \& Designed \\ Landscapes, Cuaderno de Notas y LAt. \\ Orcid ID 0000-0001-9869-8766
}

Fig. 01

Cedric Price, Two Tree Island, c. 1972. Acuarela mostrando el interior de la dársena y, detrás, viviendas de alta densidad. 
La excelente naturaleza de Two Tree Island concedía, según Price, "una oportunidad única, sobre una sólida base económica, para establecer un lugar excitante, de reposo y deleite inigualable en el Reino Unido". Esto le empujó a documentar las características ecológicas de lugar, sondear usos compatibles y diseñar un intrincado plano de zonificación ${ }^{3}$. La propuesta final incluía actividades y viviendas en relación con los distintos ecosistemas de la isla -las marismas, el rompeolas, el prado o el puerto- y una amplia variedad de tipos residenciales como carpas, caravanas, cabañas o casas-flotantes, entre otros.

Al conocerse los planes saltó la polémica y, en abril de 1972, la autoridad local de Southend, propietaria del enclave, encargó a un comité dirimir sobre el futuro de Two Tree Island. La comisión dio audiencia a una veintena de interesados y sometió las propuestas al juicio de cuarenta asociaciones y personalidades locales ${ }^{4}$. En noviembre publicó su informe final con un desenlace categórico: no consideraba ninguno de los programas y solicitaba que se garantizase la permanencia de la isla en su estado natural. Una de las siete recomendaciones del documento sintetiza su espíritu al sugerir que, "a modo de gesto nostálgico, y de acuerdo con la nomenclatura de la isla, dos árboles sean plantados sobre ella y no más".

El informe esgrimió motivos científicos para oponerse a un proyecto que, a priori, suponía la transformación de un sitio de interés ecológico. Pero, a la par, incorporó resistencias de índole afectiva, evidenciadas en su gesto nostálgico. Tales argumentos resultan previsibles si se atiende a la cultura de la conservación en Gran Bretaña, la cual nace, justamente, sobre un impulso estético previo a cualquier inquietud medioambiental ${ }^{6}$. El origen mismo del planeamiento británico estuvo en la reacción contra el crecimiento de la ciudad industrial del siglo XIX y el deseo de preservación del paisaje rural. En 1969, Peter Cowan reconocía:

"Los británicos tienen una actitud muy especial hacia su campo y su paisaje, les gusta su paisaje domesticado pero romántico, y les importa que el campo sea diseñado... han sentido que la ciudad debe ser contenida, que no se debe permitir su extensión por la faz de la nación consumiendo tierra sin control”7.

Esta actitud es remanente, tal como ha señalado J. M. Wiener, de la profunda alianza ética y estética que la cultura británica forjó con el paisaje rural en la transición hacia el siglo $X X^{8}$. Identificando el campo como alternativa única y absoluta a la reprobada ciudad industrial, se favoreció una enérgica oposición de valores: rural frente urbano, simple frente complejo, cooperación frente competencia, estabilidad frente cambio, armonía frente alienación?.

Tal desafío, a la par estético y ético, se deslizó en el fondo de las numerosas e infructuosas apelaciones que Price realizó a las autoridades tras el rechazo al proyecto. En sus escritos alegó que el plan para Two Tree Island proveía un desarrollo cuidadosamente controlado, mejoraba el carácter e interés del área y garantizaba el disfrute de la naturaleza ${ }^{10}$. Lo conseguía gracias a una intrincada composición de naturaleza, arquitectura y actividades humanas con las que, instintivamente, trataba de diluir posiciones antitéticas".

La mejor muestra de esta convicción la provee una serie de escenas paisajistas que Price elaboró para definir aspectos visuales, o más bien sensoriales, de su propuesta. Entre ellas, dieciséis postales hechas en grafito, crayón, tinta y acuarela (fig. 01). Considerando la falta 


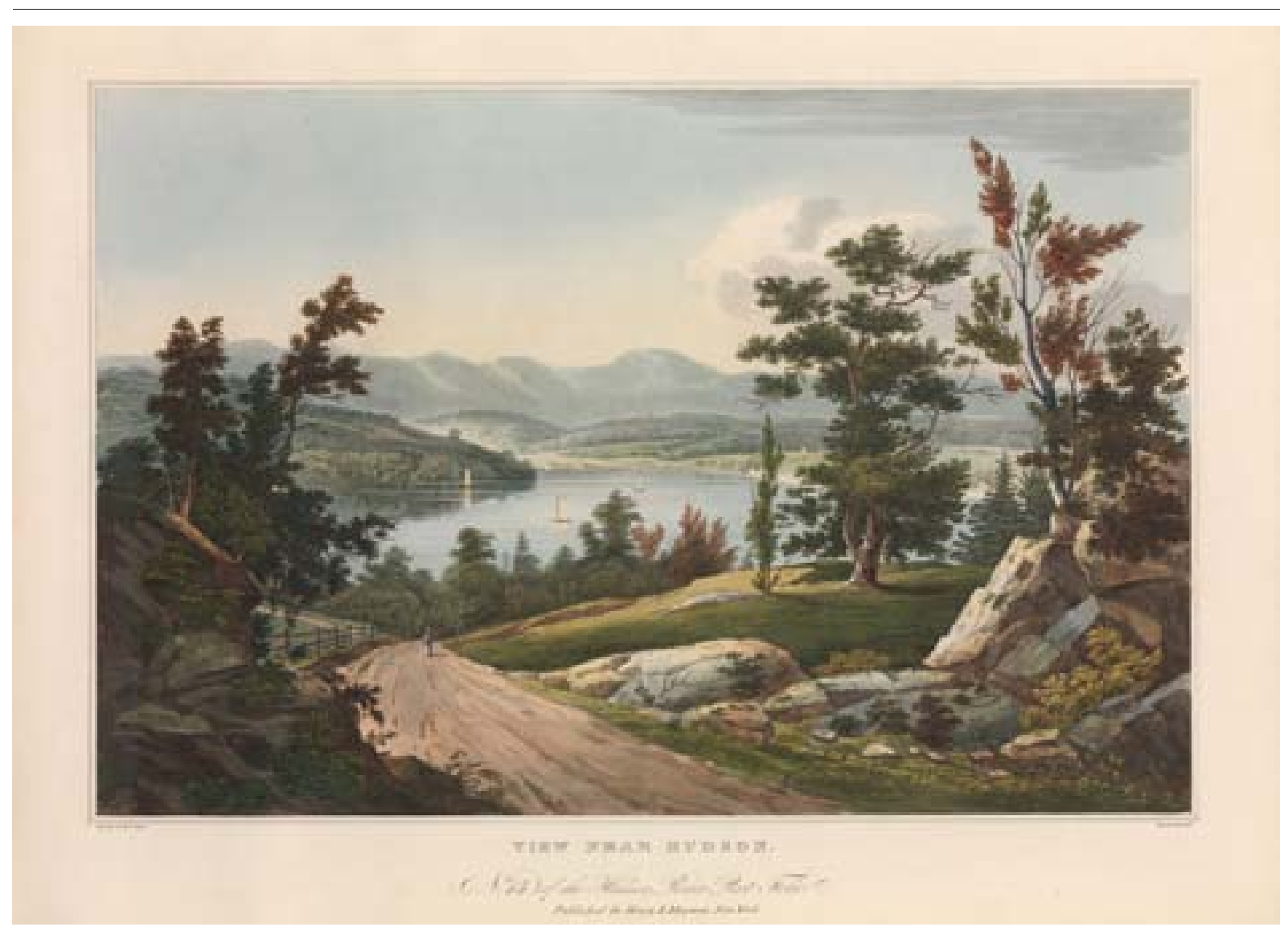

02

de definición formal del proyecto, más que configuraciones visuales precisas, las escenas definen el carácter del paisaje. Algunas se centran en la naturaleza y otras introducen el artificio fijando formas alternativas de explorar el ambiente: volar en un globo aerostático, vivir o trabajando en el agua, levantar observatorios en los árboles, y así sucesivamente.

\section{LA}

ARQUITECTURA

DEL PAISAJE

MEDIO

La controversia de Two Tree Island encarna la versión británica de una dicotomía más amplia cuya génesis fue esclarecida por el profesor Leo Marx para Norteamérica en su influyente trabajo The Machine in the Garden: Technology and the Pastoral Ideal in America (1964)'2. El autor

estudió obras notables de la literatura estadunidense que desde el siglo XVIII habían tenido por telón de fondo la incursión de la tecnología en el paisaje rural y las autorizó como lente para indagar en valores culturales hondamente asentados. Textos de Henry David Thoreau, Herman Melville, Ralph Waldo Emerson, Frank Norris, Henry Adams o Henry James le permitieron trabajar en la intersección entre literatura, ideas generales y ciertos productos de la imaginación colectiva que en conjunto denomina "símbolos culturales" ${ }^{13}$. Entre relato y relato, Marx demuestra cómo la acelerada trasformación del territorio americano había comportado enormes contradicciones de valor y significado entre su antigua estampa bucólica y su nueva imagen como potencia industrial.
Fig. 02

J. Megarey Publisher, The Hudson River Portfolio, 1820. Vista cerca de Hudson. 
Fig. 03

George Inness, The Lackawanna Valley, c. 1856. National Gallery of Art.

Fig. 04

Charles Sheeler, American Landscape, 1930. The Museum of Modern Art, Nueva York.
El libro desarrolla la coexistencia de dos modelos estéticos absolutos: jardín y máquina. El primero presta atención al paisaje como objeto de deleite, una tradición que considera importada a América por los primeros colonos europeos ${ }^{14}$. Marx relaciona su fortaleza con el jardín inglés y su cuerpo de teoría estética capaz de hacer complejas distinciones entre paisajes bellos, pintorescos y sublimes ${ }^{15}$. Este modelo determinó la primera mirada de los artistas al paisaje estadounidense, estimulando obras como The Hudson River Portfolio o Picturesque Views of American Scenery, ambas de la década de $1820^{16}$ (fig. 02).

El segundo, en cambio, es un modelo de exaltación tecnológica propiamente americano. Marx identifica su origen en la expansión del país hacia el Oeste, cuando hombre y máquina irrumpieron juntos en el paisaje selvático; la ocupación de los nuevos territorios se valió del ferrocarril como de ningún otro medio, lo que lo elevó a símbolo de progreso y fuente de inspiración artística ${ }^{17}$. Se inauguró con ello una retórica de la máquina, intensificada hasta fijar, a finales del siglo XIX, una nueva categoría estética: el sublime tecnológico ${ }^{18}$. Las nuevas imágenes hallaron en el artefacto humano aquella sensación que, hacia mediados del siglo XVIII, se reservaba a algunos accidentes y fenómenos naturales.

No obstante, la contribución de Marx no se limitó a representar ambos modelos. Su mayor conquista fue revelar cómo entre jardín y máquina prospero una tensa pero eficaz avenencia durante la segunda mitad del siglo XIX'19. The Lackawanna Valley (1855), obra del paisajista George Innes, representa el fenómeno: comisionado por el primer presidente de la Delaware, Lackawanna and Western Railroad para adelantar al público qué gloria contemplarían, su paisaje de este valle al noroeste de Pensilvania busca el balance ideal entre la celebración de la naturaleza y el novedoso entusiasmo por la tecnología ${ }^{20}$ (fig. 03). Marx lo conceptualiza como paisaje medio [middle landscape]: un modelo ponderado y estabilizado por el arte bajo la creencia de que la máquina construiría un jardín ${ }^{21}$. Solo la pérdida del equilibrio en favor de la máquina en algún momento del cambio de siglo hizo colisionar ambos modelos, fenómeno fijado por Charles Sheeler en American Landscape (1930) (fig. 04). El trabajo de Marx concluye sin resolución, cuestionando la ocasión de explorar nuevamente un paisaje medio mediante productos culturales capaces de aportar significado al ambiente postindustrial ${ }^{22}$.

En 1965, tras publicarse The Machine in the Garden, Reyner Banham contestó abiertamente a Marx reconociéndole su contribución $^{23}$. El historiador identificó la arquitectura como otro de los productos culturales que, como la literatura o la pintura, habrían contribuido a estabilizar el paisaje medio norteamericano en la segunda mitad del siglo XIX. La evidencia más plausible la encontró en The American Woman's Home (1869), donde Catherine Beecher reunía innovadoras propuestas para las casas de los nuevos territorios ${ }^{24}$. Banham recalca la formidable evolución de la autora desde su anterior libro Domestic Economy (1942), atribuyendo el hecho a la realidad de la vida y la tecnología en el Medio Oeste recién ocupado ${ }^{25}$. Identificó el esquema compositivo de Beecher, un cerramiento liviano y autónomo que envuelve un núcleo central de servicios, como el ancestro idealizado de toda casa suburbana en equilibrio de fuerzas ${ }^{26}$. A tal punto, la correspondencia de piel protectora y 


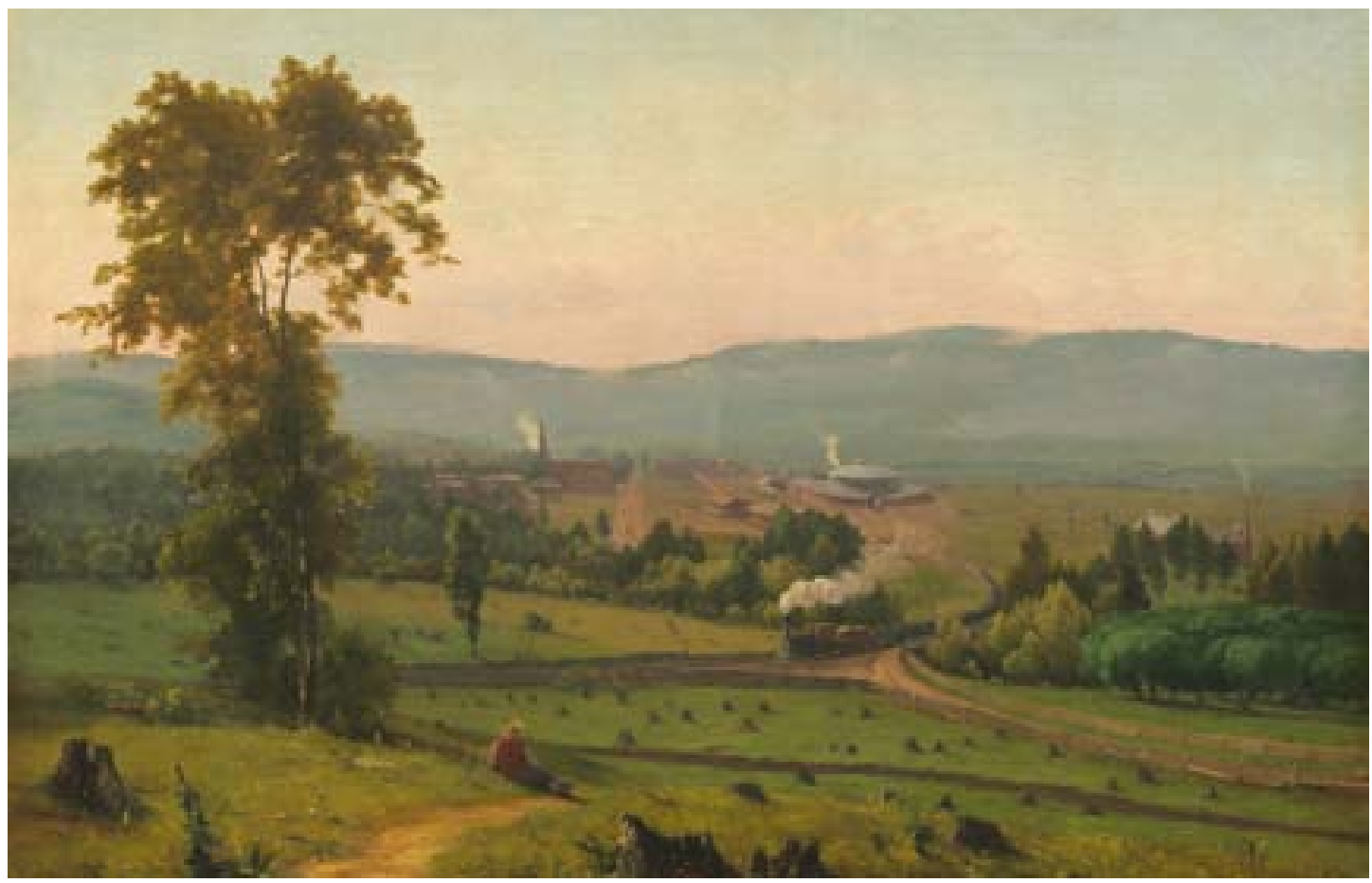

03

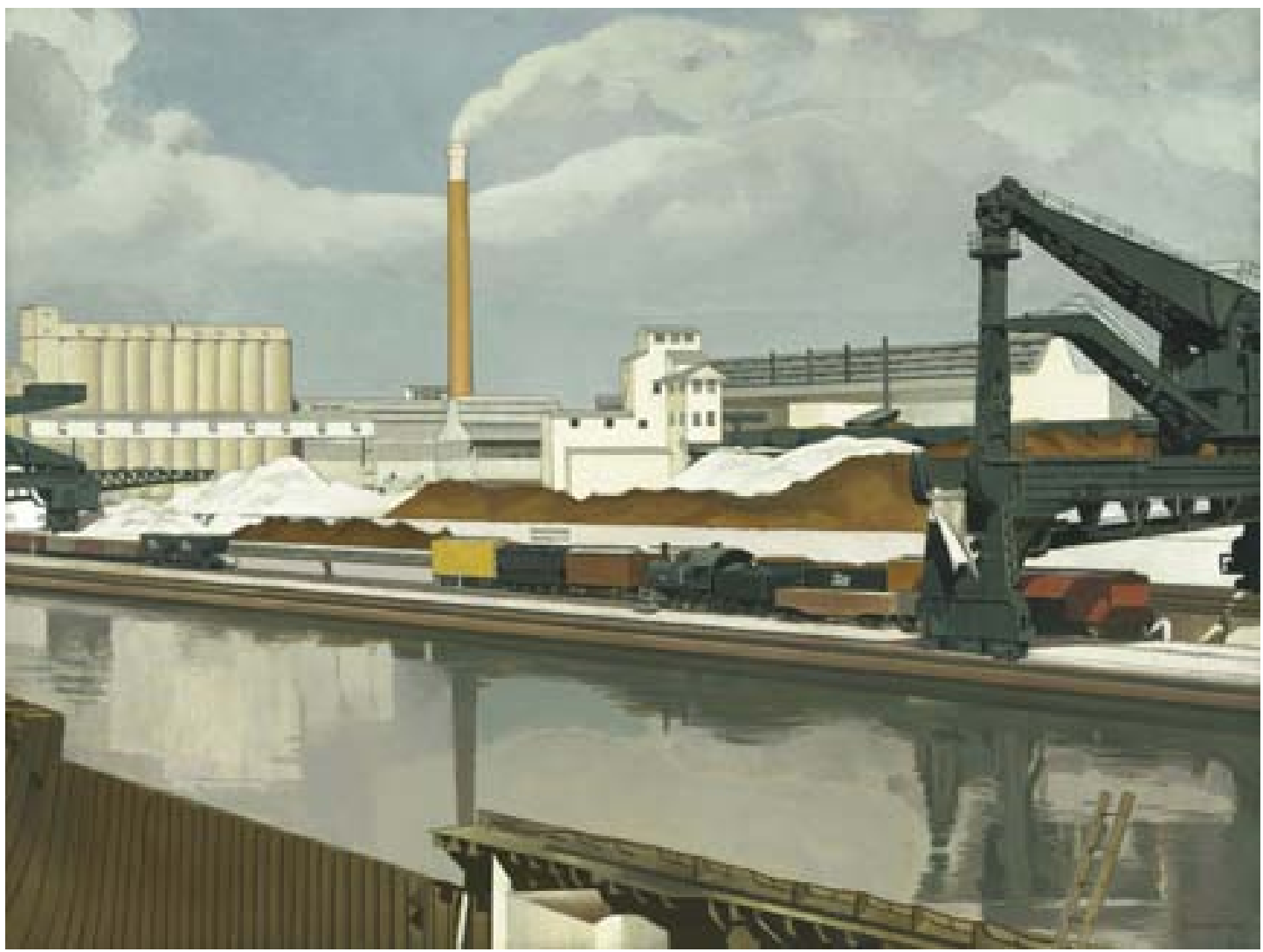


equipamiento cuantioso le permite incluso hilar una asociación histórica entre el paisaje medio y "las casas Usonianas de Wright, la casa Eames, la Glass House de Philip Johnson y la mayoría de arquitectura doméstica de los Estados Unidos que hemos admirado"27.

La génesis de esta línea de indagación formal sirvió a Banham, además, como coartada para defender en el artículo The Great Gizmo su conocido ideal de arquitectura errante: "La tecnología portátil cierra la contradicción advertida por Leo Max con tanta seguridad como hacen los significados descubiertos por los escritores célebres" ${ }^{28}$. La esencia del argumento se extiende a $A$ home is not a house, otro de sus ensayos más conocidos y también publicado en $1965^{29}$. Banham propone artefactos de vocación arquitectónica cuya descripción guarda más que un mero parecido con los principios de Beecher: además de compartir un esquema formal radiante, ambos sujetan su localización a adecuados medios de transporte y ponen en valor los beneficios morales e higiénicos del contacto con un entorno ajardinado ${ }^{30}$. Caravanas, prefabricados de fácil montaje, geodésicas, hinchables y demás sistemas de acondicionamiento ambiental transitorio, ofrecían en el presente la oportunidad de seguir la línea histórica y reeditar el paisaje medio; es decir, de resolver el enfrentamiento entre máquina y jardín.

Reencontrado el tipo arquitectónico, Banham invoca en The Great Gizmo la imagen del nuevo paisaje a construir. Lo hace eligiendo calificativos intencionados para describir actuaciones estacionales en los bosques de Connecticut, que detalla como "el paisaje suburbano quizás más paradisíaco del mundo"; y en el Medio Oeste, calificadas de "escenas que podría haber surgido del pincel de Claudio de Lorena" Encaja así la tecnología móvil en los paisajes con figuras -en las escenas pobladas o humanizadas al gusto de los pintores paisajistas del XVIII- y devuelve el ideal de paisaje al lugar donde Innes lo ubicó a mediados del siglo XIX. Es de apreciar, por tanto, lo afinado y sugerente del título con que Banham introduce su apuesta para el paisaje medio: "Landscapes with figures with gadgets".

ESCENAS DE ANALOGÍA $Y$ CAMBIO
Las ideas urbanísticas venidas desde Estados Unidos entraron con fuerza en el debate académico británico durante los sesenta ${ }^{32}$. Especial incidencia tuvo el modelo californiano. Las diferencias geográficas, históricas y culturales fundamentalmente el contraste en los ratios de uso del suelo, la relevancia de la ciudad y la apreciación del paisaje construido- obligaban a cuestionar la pertinencia de aplicar en Europa los nuevos modelos de urbanización basados en el automóvil y la muy baja densidad ${ }^{33}$. Esto no impidió el desarrollo de ideas comunes a ambos lados del Atlántico amparadas en los efectos de la globalización y el cada vez mayor parecido entre las áreas urbanas de la era postindustrial.

En ese contexto se ubica Non-Plan: An Experiment in Freedom (1969), un artículo-manifiesto firmado por Banham, Price, el editor Paul Barker y el geógrafo Peter Hall, que inspirado en la auto-organización sistémica del territorio rompe con la tradición de la planificación

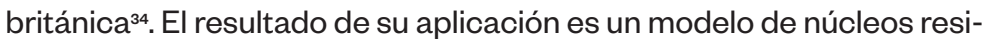
denciales menores, dispersos en el territorio pero cercanos a la ciudad, conectados con excelentes vías de comunicación, preferiblemente temporales y adaptados al nuevo "estilo de vida". Sea pues por la pretensión 
de huir de la ciudad en el caso británico, o por una condición inherente a la cultura local en el estadounidense, el suburbio se erige en lugar común de la arquitectura más experimental ${ }^{35}$.

Para estos arquitectos, panificadores y teóricos los nuevos asentamientos debían contar con una arquitectura consecuente a su condición efímera. Para ello se asumió el modelo de alojamiento a partir de un núcleo central de servicios circundado por espacios acondicionados y envueltos, a su vez, por una piel autónoma. Price avanza sobre esta cuestión en su breve ensayo Camping with Fred and James (1967), citando el esquema de tipo radiante y estableciendo variaciones en él a partir de la relación espacial que mantienen los dispositivos de acondicionamiento y las áreas servidas por estos ${ }^{36}$. Clasifica la propuesta de Banham y Dallegret, las cúpulas de Fuller y los sistemas neumáticos en función de la organización espacial que ofrecen, y discute las novedades introducidas por los robots y megaestructuras de Archigram.

Con estos antecedentes se explica por qué los británicos Cedric Price y Archigram fueron quienes mostraron más interés en rastrear el paisaje medio al modo descrito por Banham, a priori, sobre territorio americano. Sus celebradas indagaciones en busca de objetos arquitectónicos innovadores y sus menos conocidas propuestas para implantar modos alternativos de ordenación territorial se completan ahora, en torno a 1970, con escenas de paisaje que resuenan con fuerza a los paisajes con figuras de la tradición pintoresca.
Fig. 05

David Greene, Park Scene with Mobot Facilities, L.A.W.U.N. Project, 1969.

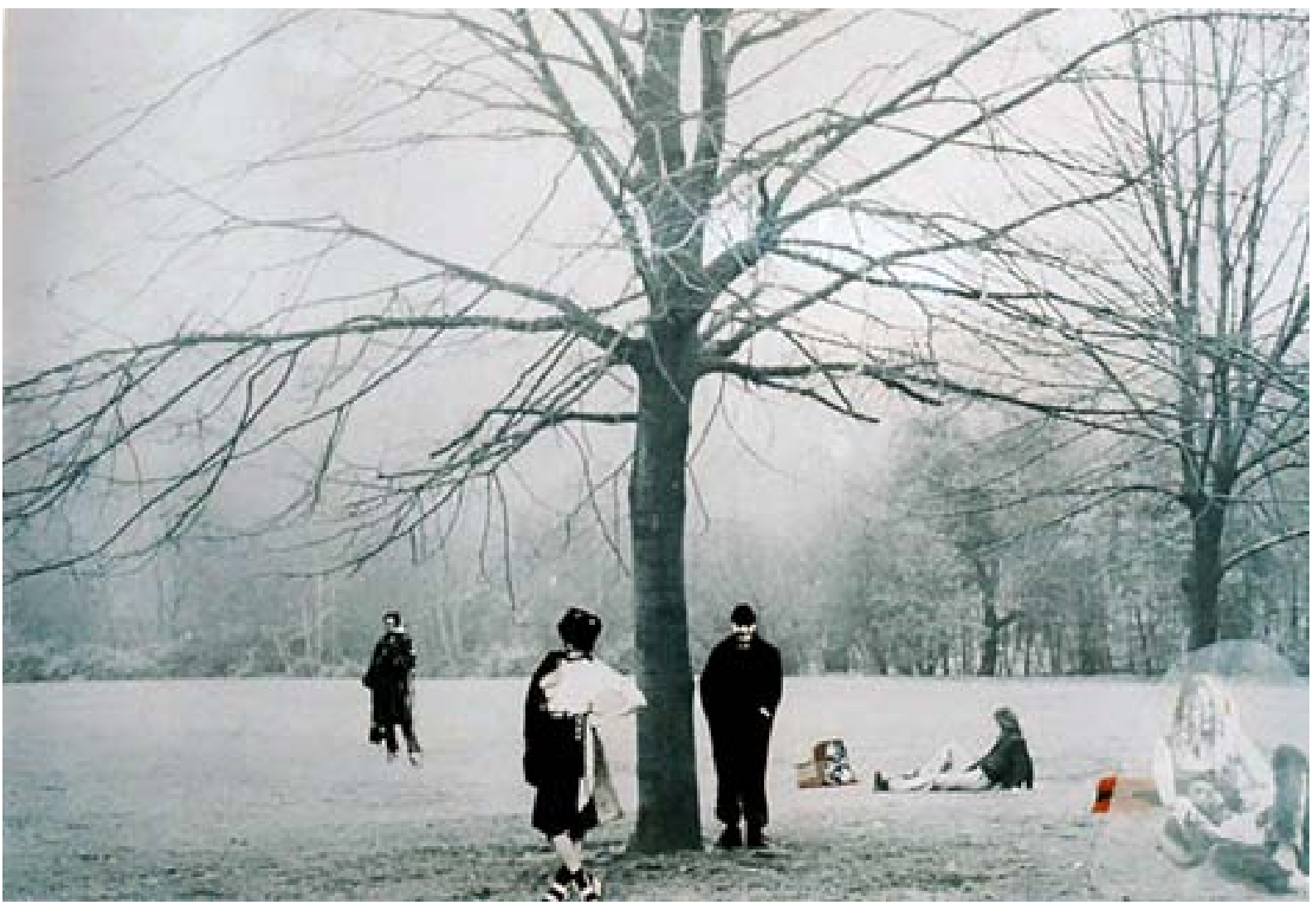


Fig. 06

Peter Cook, Settlement in a glade, Foulness Project, 1971.

Fig. 07

Cedric Price, Two Tree Island, c. 1972.

Acuarela, sin título, mostrando el aspecto interior de una zona de la isla tras la intervención.
En 1969, David Greene, miembro de Archigram, elaboró para su serie especulativa LAWUN la composición Park Scene with Mobot Facilities ${ }^{37}$ (fig. 05). Muestra individuos en un parque durante un día frío e invernal. En contraste con los abrigados paseantes, una pareja disfruta en el césped con ropa ligera. Está protegida por una membrana fina y transparente típica de la arquitectura neumática con la que por entonces experimentaban Archigram, Cedric Price o el propio Banham. Detrás, un individuo tendido en el césped parece usar un televisor portátil. El editorial de Archigram 9 calificó el trabajo de invectiva para aliviar "la tensión entre lo mecánico y lo natural" ${ }^{38}$. La palabra escena en el título, por tanto, no resulta indiferente, más aún cuando el parque muestra un aspecto muy particular. La composición remite el jardín inglés, y el título, a la construcción de la escena pictórica.

La imagen idealizada del Hedgerow Village, una aldea emplazada en algún lugar del condado de Sussex, muestra un aspecto algo más construido ${ }^{39}$ (fig. 06). Se trata de un collage elaborado por Peter Cook en 1971 que unifica con coherencia visual los hábitats de un arroyo y un lago, y al que añade recortes con vegetación para incrementar la frondosidad y diversidad cromática. En el centro se dispone la arquitectura, construida con paneles modulares, quizás desmontables, y en parte camuflada entre la vegetación, junto a un automóvil, una caravana y varias carpas. Se presentó como un modelo alternativo de suburbio en los alrededores del futuro aeropuerto de Foulness desarrollado en pequeños asentamientos ocultos en el entorno rural ${ }^{40}$.

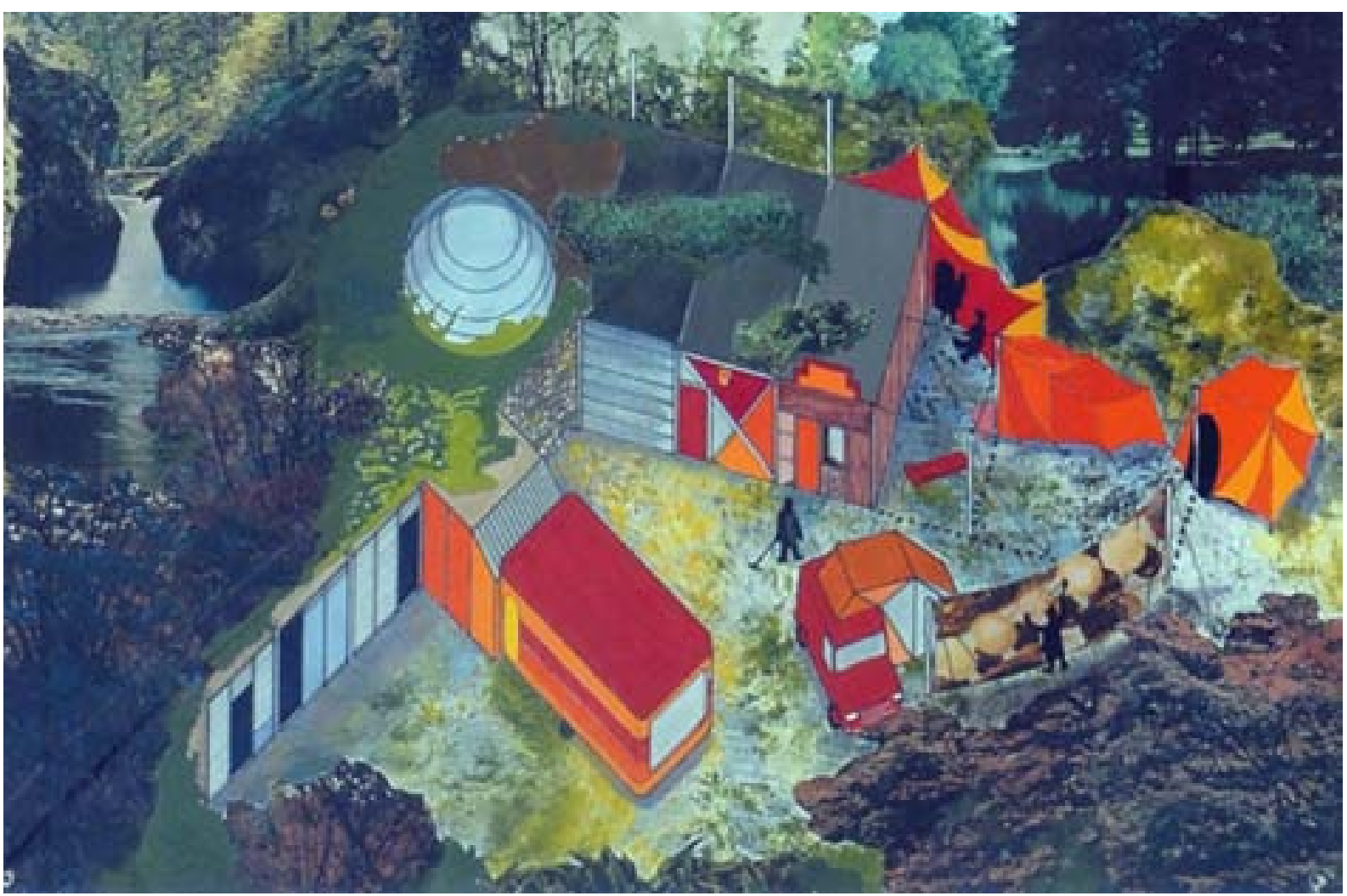




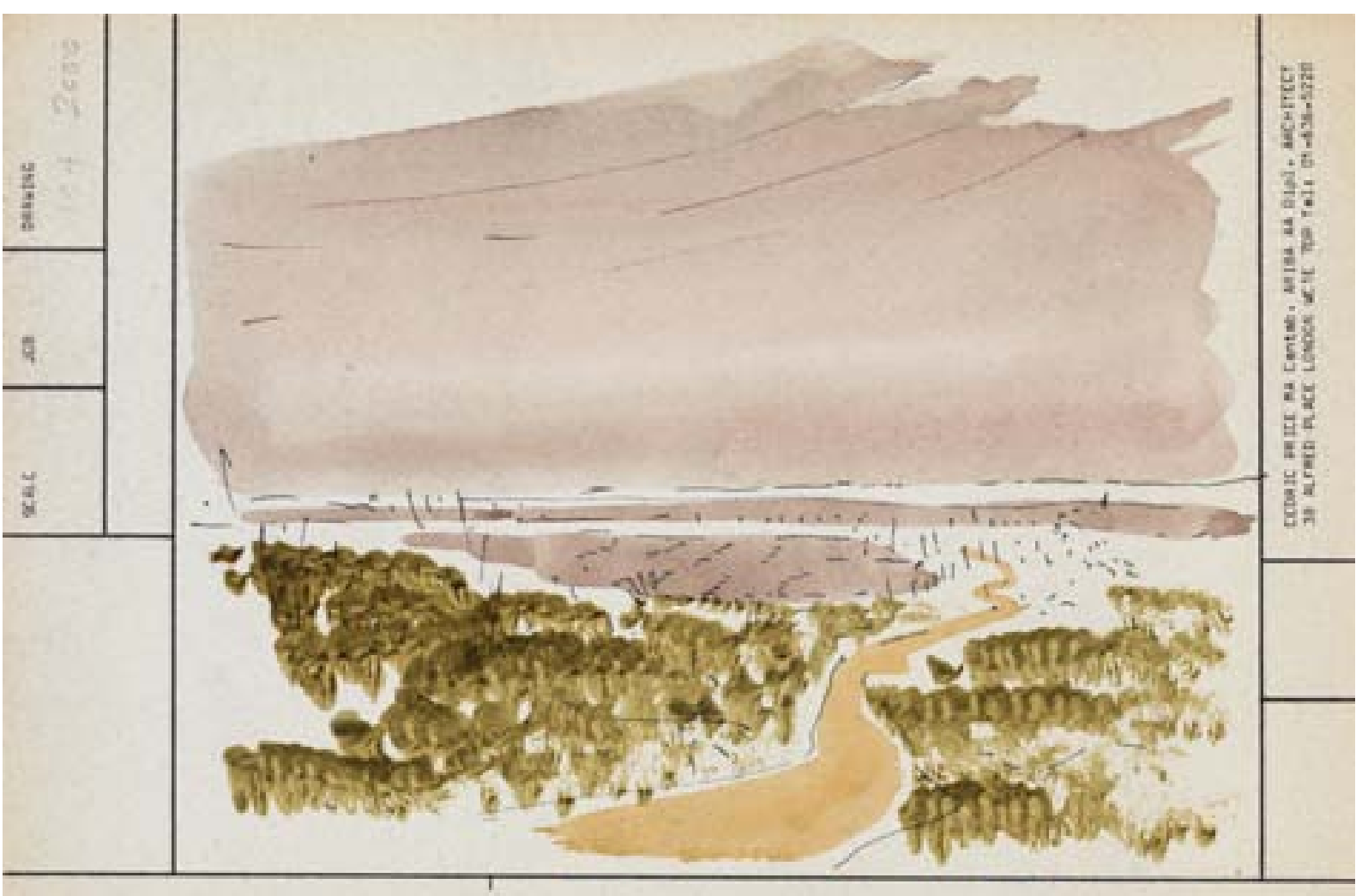

07

Como estas, Peter Cook, David Greene y Ron Herron representaron numerosas escenas de suburbios y asentamientos campestres. Para ello emplearon una diversidad de técnicas: dibujo de gran formato, collage de fotografías, impresión sobre transparencia o pintura acrílica, son algunas de ellas. La mayoría pertenecen al período 1966-1974 y ofrecen una leve desviación de la línea dominante en las propuestas del grupo hasta entonces. Las megaestructuras se fragmentaron en tecnología y robots de acondicionamiento temporal, permitiendo definir una versión propia de lo que debía o podía ser el paisaje británico postindustrial. Una actitud tendente a la desintegración del objeto arquitectónico que Hadas Steiner ha calificado como el "pintoresco tecnológico" de Archigram ${ }^{41}$.

También Cedric Price elaboró escenas paisajistas desde 1967, apareciendo las primeras en sus proyectos Plotteries Thinkbelt, Port Eliot \& Port Hole y Atom ${ }^{42}$. Pero es a partir de 1969 cuando surgen las más características, como la citada serie para Two Tree Island y las que acompañan a sus proyectos en Glasgow y Abu Dabi, así como el más tardío Generator ${ }^{43}$. El progreso en estas escenas viene dado por una menor atención al objeto arquitectónico en favor del intrincamiento entre artificio y naturaleza, aumentando además el uso de color. Revelan cierta especialización al emplearse repetidamente tarjetones apaisados de veintidós por treinta centímetros con el layout del estudio, sobre los que aplica técnicas ágiles (fig. 07). No obstante, Price ofrece variantes, como collages y sencillos dibujos sobre fotocopias de fotografías, una práctica especialmente efectiva para poner en valor artefactos preexistentes. En 
general su técnica es menos elaborada que la preferida por los miembros de Archigram, obteniendo representaciones más ajustadas al canon tradicional pero no por ello menos significativas.

Varias postales de este género elaboradas por Price en 1973 muestran el horizonte idealizado de la playa de Abu Dhabi una vez construido el Jardín Marino, una gran barrera de protección de la costa dotada de equipamiento para el ocio ${ }^{44}$. Un funicular marítimo, pasarelas entre coral, jardines subacuáticos, cúpulas de observación, tribunas flotantes para eventos o boyas móviles para ordenar la superficie del mar, son ejemplos de la tecnología que dirige al usuario a descubrir la bahía artificial a través de nuevas formas de percepción. El horizonte marcado en las postales está en plena evolución: los rayos del sol, las fuentes, la brisa, el movimiento de los barcos (fig. 08). Con una mezcla de pluma, gouache y ceras, Price estudió el dinamismo del conjunto, incluida la exhibición de agua, luz y color cuando, una vez al día, se activaban surtidores y fuentes en la bahía artificial.

Otra serie de ocho escenas fue realizada ya en la segunda mitad de los setenta para Generator, un centro de actividades y descanso emplazado provisionalmente en un entorno rural entre los estados de Georgia y Florida ${ }^{45}$ (fig. 09). Price parte de dos croquis del entorno natural donde teóricamente se ubicaría el proyecto, mirando al noreste y suroeste del sitio, y los fotocopia para elaborar una variedad de escenas. Los panoramas imbrican naturaleza y artificio, y evocan los cambios y contingencias del paisaje construido sobre un mismo emplazamiento, dando representaciones más o menos figurativas. Puntos radiantes, planos de luz o composiciones con elementos fluorescentes son algunos de sus recursos para evocar las sensaciones del cuerpo inmerso en el paisaje dinámico.

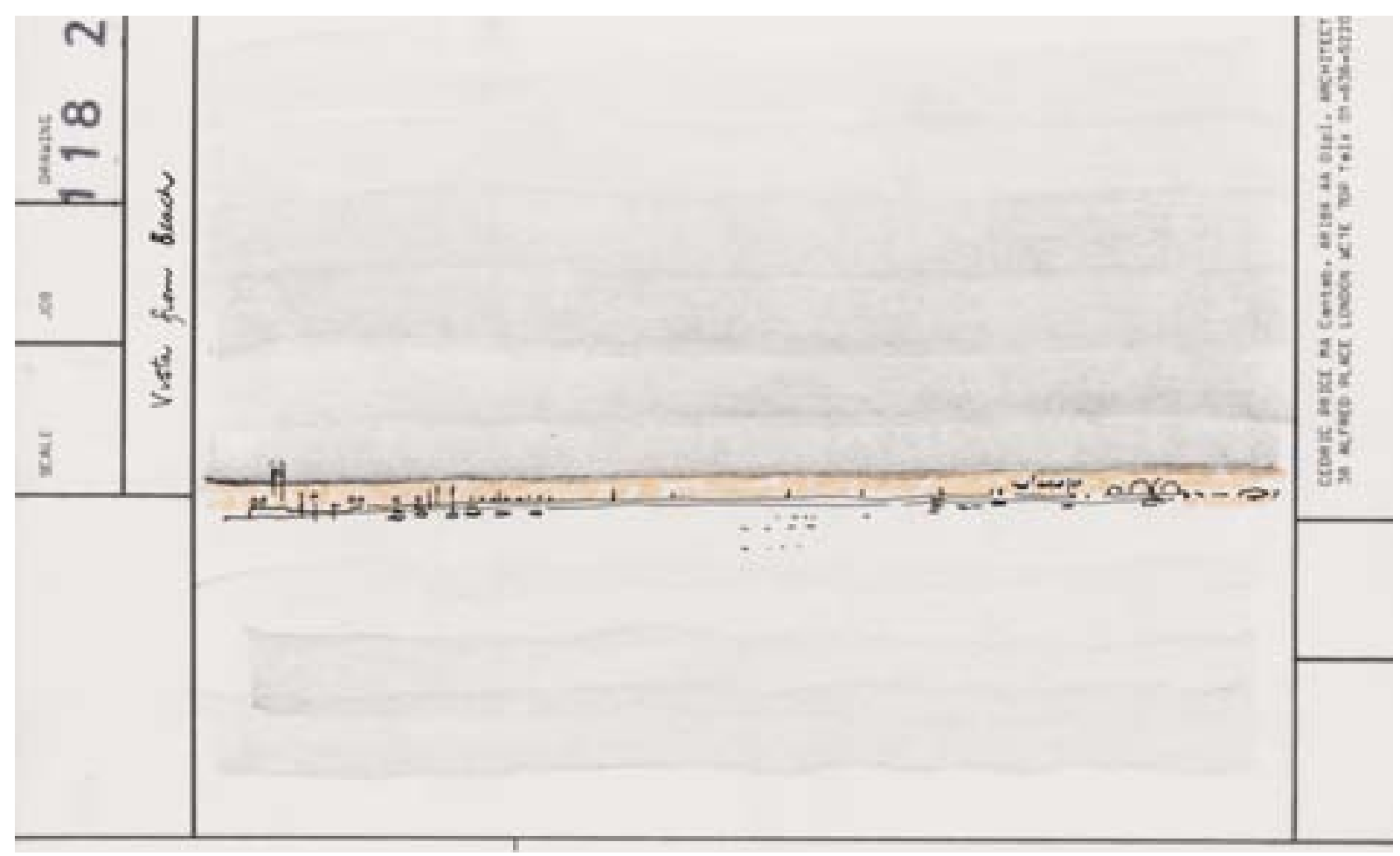


Una variante en la técnica es su propuesta para un concurso de ideas promovido por la ciudad de Glasgow en 1972 con el objetivo de regenerar el área industrial obsoleta el río Clyde ${ }^{46}$. Price elabora una colección de veintidós pequeñas escenas manipulando fotocopias de fotografías del estado actual sobre las que dibuja libremente con vivos colores (fig. 10). La técnica le permite incorporar a la composición los grandes artefactos en ruina y tratar cielo, río y tierra firme. Destaca su propensión a introducir césped y vegetación dotando al área del carácter de un parque, pero que no solo cumple una función ornamental: la plantación podría estar en tierra, dotada de sistema hidropónico, o flotar sobre la superficie del río pero, como en el campo, combinando productividad y placer estético.

LA CONDICIÓN PINTORESCA EN LA

ARQUITECTURA EXPERIMENTAL
Tomando en consideración la línea histórica trazada por Banham, estos paisajes no pueden ya interpretarse como una novedad radical y rupturista, sino alineados con una tendencia histórica válida. Los trabajos mencionados llevan al pintoresco británico a una nueva fase,

análoga, en parte, a lo que había sido el paisaje medio estadunidense. Es decir, las escenas introducen variaciones sobre el pintoresco tradicional al tratar de imponerle aquellas cualidades que habían emergido espontáneamente en Norteamérica a mediados del siglo XIX. Así, el acceso a los asentamientos suburbanos representados requiere de la expansión de efectivas redes de transporte y su arquitectura se puede clasificar en tipos residenciales de esquema radiante formados por núcleo de servicios y piel autónoma. Las propuestas, por

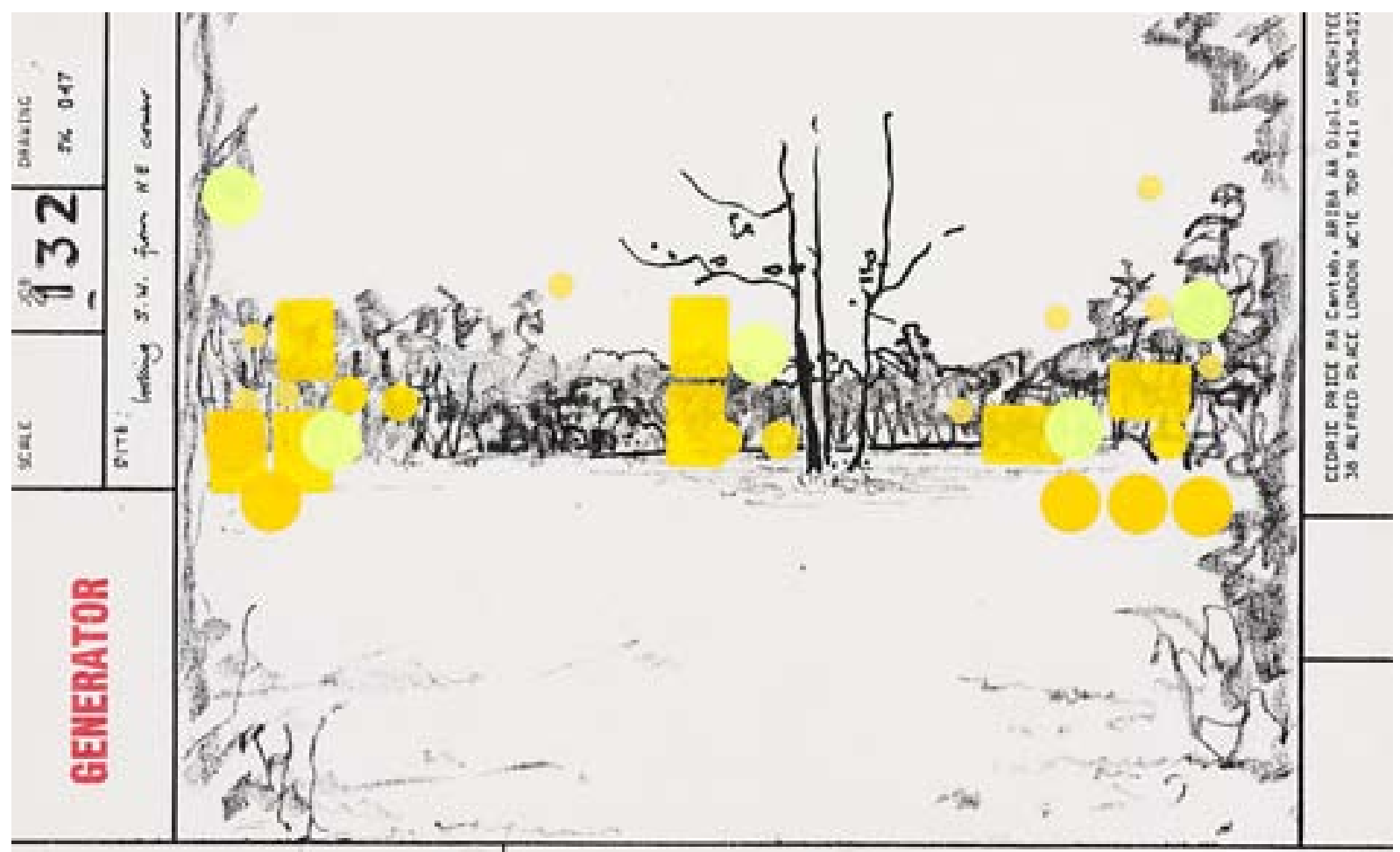


tanto, asumen las características básicas que Banham identifica en el paisaje medio y que pretende reproducir en paisajes con figuras con gadgets.

La primera variación introducida sobre la tradición del pintoresco británico es, justamente, la organización de una dialéctica entre el paisaje rural, como lo bello, y la tecnología, como la depositaria de la experiencia renovada de lo sublime. En la estética pintoresca se admitió tradicionalmente una síntesis dialéctica entre belleza pastoril y paisajes sublimes de la naturaleza. Gilpin reconoció belleza pintoresca, de hecho, en aquellas pinturas en las que los cambios y rugosidades de la escena posicionaban a la obra en un estadio intermedio entre lo bello y lo sublime. Al reconocer lo pintoresco como categoría, Uvedale Price permitió su individuación como tercera opción, pero no por ello dejó de reconocerle su capacidad mediadora. Así, sobre la necesidad de definir el par formado por lo bello y lo sublime para conseguir lo pintoresco, se introduce la vía de renovación: los nuevos paisajes continúan combinando naturaleza y artificio, pero el poder estético del artefacto ha sido renovado. Ahora, la arquitectura neumática, transformable o móvil adquiere un rol superior al asignado al artificio en las escenas pintorescas, porque encarna el sublime tecnológico.

Por otro lado, las escenas evidencian que el aristócrata solitario que pasea a través de sus fastuosas posesiones, vinculando el jardín con la propiedad y la vida privada, ha sido sustituido por el ciudadano de un Estado democrático ${ }^{47}$. Esto, en palabras de Banham, era una condición inherente al propio ruralismo americano nacido liberal, pero no del europeo, dado su origen feudal: frente al patrón urbano y social preexistente en Europa, Estados Unidos no tenía ni sociedad ni propiedad de la tierra hasta la llegada del ferrocarril, que introdujo el primer patrón de orden humano en su territorio. Para los experimentales británicos, la superación de esta diferencia la facilitaban los profundos cambios en el estilo de vida del país en aspectos como la familia, la educación, el trabajo y el tiempo libre, con consecuencias directas sobre la movilidad, el turismo y el ocio ${ }^{48}$. Por ende, los ambientes pintorescos representados en las acuarelas de Price para Two Tree Island y en los montajes de parques de Greene no son una invención accidental, sino demandas sociales de su tiempo identificadas como oportunidad para la arquitectura. Otra condición del renovado pintoresco tiene que ver, por tanto, con la democratización de su experiencia estética.

A finales del siglo XVIII, Uvedale Price había escrito, superando a la mayor parte de los teóricos que circunscribían el género pintoresco a lo visual -a pensar el mundo exterior en términos de perspectivas-, que el efecto general de lo pintoresco "es producir algún tipo de deleite o placer para el ojo, para la imaginación, o para ambos"y que "el espíritu tiene que ser estimulado tanto como aliviado"49. En general, los nuevos paisajes continúan a la búsqueda del placer estético basado en la estimulación del psicologismo y la imaginación -en el "placer kinestésico", en palabras favoritas de Price. Pero la duración de la experiencia estética es ahora considerada en su continuidad temporal y espacial: frente a su confinamiento en el jardín, se propaga al paisaje cotidiano ${ }^{50}$. La experiencia estética activa que atañe al estímulo de la imaginación, y liga conocimiento y emoción, memoria y descubrimiento, muestra aquí su ideal: es un acto de conocimiento a través de lo sentido. 
Asimismo, las nuevas escenas del paisaje medio no pueden ser leídas en su configuración visual de la misma manera que la sucesión de vistas en el jardín-circuito o los croquis de sus coetáneos del townscape ${ }^{51}$. Resultaría imposible, dada la indeterminación de los proyectos. Si el jardín inglés toma la naturaleza como modelo, ahora el proceso da un paso más, ya que las escenas, como imaginario, tratan de buscar exasperadamente un modelo de referencia para la imagen de la naturaleza en el tiempo postindustrial. Ocupan un lugar delantero, como medio expresivo que hace de guía, estimulando la imaginación a través de la memoria y de los enigmas fenomenológicos inherentes al propio paisaje. Como en el pasado, distinguen un paisaje dotado de cualidades pictóricas, pero a su vez, proyectan un paisaje que no existe y merece, necesita, ser construido. RA
Fig. 10

Cedric Price, River Clyde Competition, c. 1973. Vistas de algunas áreas de proyecto cubiertas con vegetación asociada a usos ornamentales, deportivos y productivos.
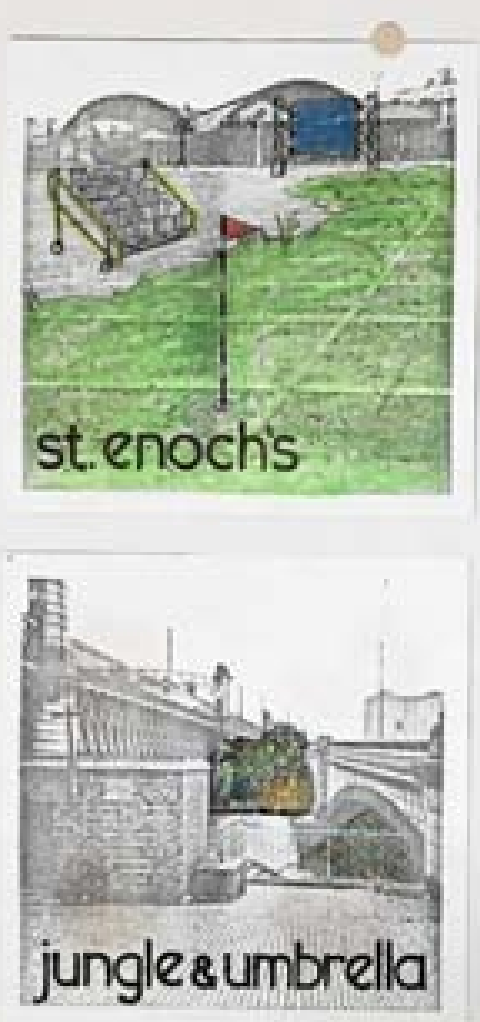
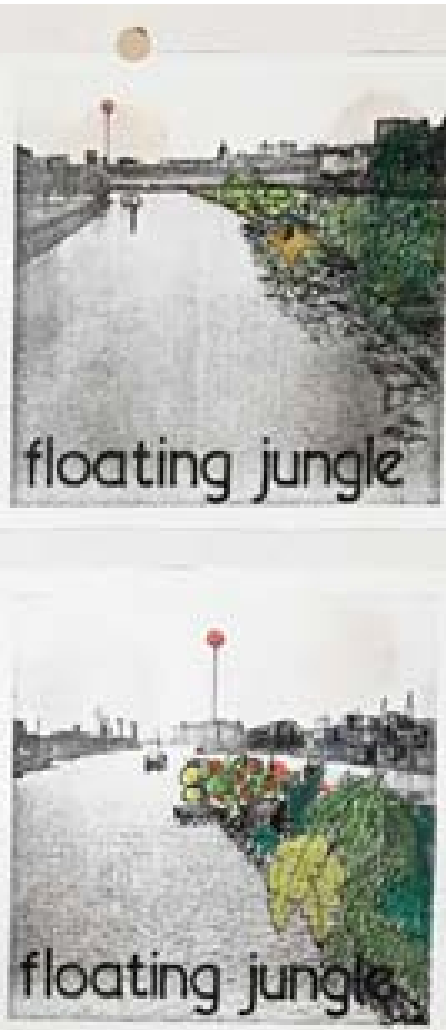
01. El proyecto apareció publicado en el monográfico editado por el propio autor, PRICE, Cedric, Cedric Price: Works II, Architectural Association, Londres, 1984, pp. 86-87. Una reseña más extensa aparece en HARDINGHAM, Samantha, Cedric Price works 1952-2003: a forward-minded retrospective, Architectural Association \& Canadian Centre for Architecture, Londres/ Montréal, 2016, pp. 359-363.

02. CPA \& YRMA, Two Tree Island Project for David Keddie Consultant's Report, Londres, febrero 1972. Documento del fondo Cedric Price, Canadian Centre for Architecture, Montréal.

03. DE LA O CABRERA, Manuel Rodrigo, "Planning for enabling: an analysis of Cedric Price's proposal for Two Tree Island, 1971-1973", en Planning Perspectives, 2017, pp. 1-22.

04. COUNTY BOROUGH OF SOUTHENDON-SEA, Report of Two Tree Island and Leigh Marshes Development Sub-Committee of the Policy and Finance Committee, noviembre de 1972. Documento del fondo Cedric Price, CCA, Montréal.

05. Ibid. p. 9.

06. MATLESS, David, Landscape and Englishness, Reaktion Books, Londres, 1998, pp. 25-42.

7. COWAN, Peter, "Introduction", en COWAN, P. (Ed.) Developing Patterns of Urbanisation, Oliver \& Boyd, Edimburgo, 1970, p. 3. Renombrado geógrafo, Peter Cowan fue director de la Joint Unit for Planning Research del University College London. A partir de 1967, Price se integró junto a otros académicos en un grupo de trabajo liderado por Cowan sobre nuevos patrones de urbanización en el Centre for Environmental Studies. Existe una clara coincidencia entre la agenda de Cowan y los temas discutidos por Price en sus artículos y proyectos de esta época. Sobre las afiliaciones de Cedric Price en las discusiones de planificación a fines de la década de 1960. Ver DE LA O CABRERA, M.R., op. cit, pp. 4-7.

08. WIENER, J. Martin, English culture and the decline of the industrial spirit 18501980, Penguin, Londres, 1981.

09. Ibid. pp. 157-159.
10. Price se apoyó en dos documentos gubernamentales, Leisure in the Countryside y Planning for Leisure. Ambos supusieron un cambio en las políticas de planificación y conservación del campo británico al reconocer la necesidad de naturaleza de los habitantes de las ciudades y vincular ocio, agricultura y ecología. Ver SILLITOE, Kenneth K., Planning for leisure, H.M.S.O., Londres, 1969 , y CMND 2928, Leisure in the Countryside of England and Wales, H.M.S.O., Londres, 1967.

11. DE LA O CABRERA, M.R., op. cit., pp. 15-18.

12. MARX, Leo, The Machine in the Garden: Technology and the Pastoral Ideal in America, Oxford University Press, Nueva York, 1964. Ver el análisis de CANNAVO, Peter F., "American contradictions and pastoral visions: An appraisal of Leo Marx, The Machine in the Garden", Organization \& Environment, marzo 2001, vol. 14, n. 1, pp. 74-92.

13. BRYANT, John L. "A Usable pastoralism: Leo Marx's method in the machine in the garden", en American Studies, primavera 1975, vol. 16, n. 1, pp. 63-72.

14. MARX, L., op. cit., pp. 88-89.

15. Íbid. Marx se apoya, sin citarlo, en el libro de HUSSEY, Christopher, The picturesque: studies in a point of view, Frank Cass \& Co, Londres, 1927, reeditado en 1967.

16. Sobre el proceso cultural de apreciación del paisaje norteamericano a través de la pintura y la literatura influída por la teoría estética del jardín inglés, ver FITCH, Jamers Marston, "American Pleasure Garden", en Architecture and the Esthetics of Plenty, Nueva York, Columbia University Press, 1961, pp. 180-187.

17. El ferrocarril de pasajeros apareció en la escena estadounidense por vez primera en la década de 1830, y casi de inmediato llamó la atención de varios de los pintores paisajistas más célebres, como Thomas Cole, Thomas Doughty, Asher Durand y John Kensett. Ver MARX, L. "The Railroadin-the-Landscape: An lconological Reading of a Theme in American Art", en DANLY, Sy MARX, L. (Eds.), The Railroad in American Art: Representations of Technological Change, Cambridge, MIT Press, 1988, pp. 183-208.
18. MARX, L., op. cit., pp. 195

19. Ibid. p. 226.

20. CIKOVSKY, Nicolai, "George Inness and the Hudson River School: 'The Lackawanna Valley"', en American Art Journal, 1970, vol. 2, n. 2, pp. 36-57.

21. MARX, L., op. cit., pp. 228-229.

22. Ibid. pp. 364-365

23. REYNER, Reyner, "The Great Gizmo", en Industrial Design, 1965, n. 12, pp. 58-59.

24. The American Woman's Home está en realidad firmado por dos hermanas, Catherine y Harriet Beecher. Banham considera este libro a partir del análisis realizado por FITCH, J. M., "Our Domesticated Utopians", en Architecture and the Esthetics of Plenty, pp. 65-85. Fitch se refiere a la ubicación de la casa en el paisaje: "the house sit on spacious plots and the whole family gardens, but now one feels that the motive is as much moral as economic", p. 75.

25. BANHAM, R., Architecture of the Well-Tempered Environment, University of Chicago Press, Chicago, 1969, p. 102.

26. BANHAM, R., "The Wilderness Years of Frank Lloyd Wright", en A Critic Writes: Selected Essays by Reyner Banham, University of California Press, Berkeley, 1996, pp. 137-151. Originalmente publicado en RIBA Journa, 1969, n. 76, pp. 512-518.

27. Ibid., pp. 139-140.

28. BANHAM, R., “The Great Gizmo”, p. 58.

29. BANHAM, R., "A Home is not a House", en Art in America, 1965, n. 2, pp. 109-118. En el artículo Banham presenta una propuesta de arquitectura neumática desarrollada junto a François Dallegret.

\section{Ver nota 24}

31. Ibid.

32. COWAN, P., op cit, p. 4, señaló: "Y si hay profundas diferencias entre las dos culturas en sus actitudes hacia la urbanización, ¿estamos en lo correcto al tomar a los Estados Unidos occidentales como un modelo para el futuro de este país?". Ver cita 7. 


\section{Referencias bibliográficas}

33. Los argumentos de Banham en The Great Gizmo y de Cowan en su introducción a Developing Patterns of Urbanisation coinciden al señalar las diferencias territoriales, históricas y culturales entre ambos países.

34. BANHAM, R., BARKER, P., HALL, P., PRICE, C. Non-Plan: An Experiment in Freedom, en New Society, 1969, n. 338, pp. 435-443.

35. Si el ruralismo británico del siglo $X X$ surgió como antídoto idealizado a los males reales de las ciudades, el norteamericano se revelaba como la esencia misma de una nación capaz de mantener "una cultura creativa y llena de vida sin ciudades". Ver BANHAM, R, "The Wilderness Years of Frank Lloyd Wright", p. 138

36. Price, C. Camping with Fred and James, en Architectural Design, March 1967, p. 106.

37. GREEN, David, “Gardener's Notebook: LAWUN Project Number One", en Architectural Design, 1970, n. 8, pp. 385-387. Un año antes apareció en GREEN, David, "Gardener's Notebook", Architectural Design, 1969, p. 507.

\section{Archigram, n. 9, 1970, p. 1.}

39. COOK, Peter, et al., en Archigram, Studio Vista, Londres, 1972

40. No por casualidad, Two Tree Island también se emplaza en el condado de Essex y la futura construcción del aeropuerto de Foulness fue una de las razones estratégicas que llevó al empresario local David Keddy a promover el proyecto.

41. STEINER, Hadas A., Bathrooms, Bubbles and Systems: Archigram and the Landscapes of Transience, $\mathrm{PhD}$ Dissertation, Massachusetts Institute of Technology, 2001. Ver también STEINER, H. A., Beyond Archigram: the structure of circulation, Routledge, Nueva York, 2009, pp. 222-242. Steiner ha conceptualizado como pintoresco tecnológico la tendencia de Archigram a representar entornos efímeros en los que el artefacto se funde en el lugar. Añade que, si bien el pintoresco tradicional presentaban naturaleza y cultura como indistinguibles, el de Archigram presentó la tecnología como el paisaje inextricablemente entrelazado con la arquitectura. Además, en lugar de un conjunto estable de formas, el pintoresco tecnológico vendría determinado por proyecciones de deseo.

42. HARDINGHAM, S., op. cit., pp. 192-207, 230-237, 242-253. Más escenas se pueden encontrar en PRICE, Cedric, op. cit., pp. 24-25, 27-29, 60, 86, 91, 95. En referencia al proyecto Plotteries Thinkblet, Abalos ha identificado "genuina inspiración pintoresca" en la amalgama entre nuevas construcciones y ruinas industriales. Ver ABALOS, Iñaki, Atlas pintoresco, Vol. 2: Los Viajes, Gustavo Gili, Barcelona, 2008, p. 214

43. HARDINGHAM, S., op. cit., pp. 358-365.

44. DE LA O CABRERA, M.R., "'Kinaesthetic delight': Cedric Price's plan for the Abu Dhabi Sea Garden, 1973", Studies in the History of Gardens \& Designed Landscapes, 2017, vol. 37, n. 3, pp. 250-260.

45. Ibid. pp. 446-469. PRICE, Cedric, op. cit., pp. 91, 95 .

46. Ibid., pp. 406-413.

47. PRICE, Cedric, "Public spaces and private spaces", en London Architecture Club Magazine, 1978, n. 2, pp. 20-22.

48. WILLMOTT, Peter, "Some Social Trends", Urban Studies, 1969, vol. 6, n. 3, pp. 286-308.

49. PRICE, Uvedale, An essay on the picturesque, as compared with the sublime and the beautiful: and, on the use of studying pictures, for the purpose of improving real landscape, James Robson Publisher, Londres, 1796, pp. 166, 235.

50. PRICE, Cedric, "Approaching an Architecture of Approximation", Architectural Design, septiembre 1972, n. 10, pp. 645-647.

51. Sobre el empirismo visual en la época, ver MACARTHUR John, AITCHISON Mathew, "Oxford versus the Bath Road: empiricism and romanticism in The Architectural Review's picturesque revival", The Journal of Architecture, 2012, vol. 17, n. 1, pp. $51-68$.
- COWAN, Peter (Ed.), Developing Patterns of Urbanisation, Oliver \& Boyd, Edimburgo, 1970.

- DANLY, S y MARX, L. (Eds.), The Railroad in American Art: Representations of Technological Change, Cambridge, MIT Press, 1988.

- DE LA O CABRERA, Manuel Rodrigo, "Planning for enabling: an analysis of Cedric Price's proposal for Two Tree Island, 19711973", en Planning Perspectives, 2017.

- FITCH, Jamers Marston, Architecture and the Esthetics of Plenty, Nueva York, Columbia University Press, 1961.

- GREEN, David y HARDINGHAM, Samantha, L.A.W.U.N Project 19: The Disreputable Projects of David Greene, AA Publications, Londres, 2008.

- HARDINGHAM, Samantha, Cedric Price works 1952-2003: a forward-minded retrospective, Architectural Association \& Canadian Centre for Architecture, Londres, 2016.

- MARX, Leo, The Machine in the Garden: Technology and the Pastoral Ideal in America, Oxford University Press, Nueva York, 1964

- MATLESS, David, Landscape and Englishness, Reaktion Books, Londres, 1998.

- PRICE, Cedric, Cedric Price: Works II, Architectural Association, Londres, 1984.

- REYNER, Banham, A Critic Writes: Selected Essays by Reyner Banham, University of California Press, Berkeley, 1996

- STEINER, Hadas A., Beyond Archigram: the structure of circulation, Routledge, Nueva York, 2009.

- TEYSSOT, Georges, "The American lawn: the spectacle of suburban pastoralism", en Lotus international, 1999, n. 101, pp. 92-115.

- WIENER, J. Martin, English culture and the decline of the industrial spirit 1850-1980, Penguin, Londres, 1981.
RA. Revista de Arquitectura Núm. 20 - 2018 P. $70-85$ 